in the field. It is based primarily on human material, reflecting the tremendous growth that has occurred in this body of data during recent years. Structural chromosome abnormalities pose challenging problems for the cytogeneticist and the clinician alike. Balanced rearrangements can give rise to abnormal offspring and are a significant cause of spontaneous abortion and infertility. Furthermore, acquired autosomal rearrangements are now recognised as a major component of the neoplastic process and, as the nature of the associated molecular events gradually unfolds, are becoming increasingly useful in the diagnosis and management of many malignant conditions. At the other end of the spectrum, the role of autosomal rearrangements in promoting population diversification and speciation, by creating barriers to panmixia, has long been recognised.

The book is divided, rather arbitrarily perhaps, into four sections. The first deals with the meiotic consequences of balanced translocations, pericentric and paracentric inversions, and insertions. Both livebirth and amniocentesis statistics are examined and empirical models of risk for the production of viable unbalanced progeny are proposed. It appears that the identities of the chromosomes involved, the length and nature of the segment in imbalance, the effect of trisomy compared with monosomy, the type of segregation, the pachytene configuration, the mode of ascertainment, and the sex of the carrier can all influence the degree of risk. Regrettably though, despite a degree of overlap in the subject matter of these chapters, a consensus view on the relative importance of these risk factors does not emerge. In the second section contributors explore the effects of translocations in man, domestic animals, and rodents on gametic selection, fertility, and reproductive loss. The potential scale of this problem is highlighted by the elegant technique of karyotyping human spermatozoa, which shows that up to $77 \%$ of sperm from carriers of autosomal rearrangements can have an unbalanced complement. The penultimate section on the origin of chromosome rearrangements treats the subject from both evolutionary and aetiological standpoints. First, the importance of chromosome fusion and fission in mammalian karyotypic diversification is evaluated, then unbalanced structural abnormalities which usually occur de novo are examined; stable dicentrics, isochromosomes, rings, duplications, and the intriguing and incorrectly named anomaly, inv dup(15), are described in some detail. A fascinating study using heterochromatic variants shows that in a reversal of the situation in Down's syndrome $84 \%$ of such de novo rearrangements are paternal in origin. The last section in this book reflects on the significance of breakpoints in chromosome abnormalities and contains a diverse and somewhat disjointed collection of nonetheless interesting chapters, which include phenotype/karyotype correlations in man and mouse, small deletion syndromes associated with Mendelian disorders, the incidence and effects of mobile genetic elements, cancer cytogenetics, and gene mapping.

This is an excellent, well presented book, bringing together a wealth of information that would otherwise only be available from a variety of quite different sources. It should be essential reading for every cytogeneticist and it should prove very useful for researchers, despite being slightly out of date. In containing wide ranging material, it will no doubt, as the editor indeed contends, contribute to the cross fertilisation of ideas within this field. The busy clinician, however, might find that a few of the articles are peripheral to clinical interest and that much of the more valuable information on risk assessment is impossible to access quickly.

SELWyN H RobERTS

\section{Duchenne Muscular Dystrophy}

Revised edition. Oxford Monographs on Medical Genetics No 15. By Alan E H Emery. (Pp 317; £17.50.) Oxford: Oxford University Press. 1988.

This revised paperback edition of Alan Emery's excellent book on Duchenne muscular dystrophy contains an enlarged chapter on molecular pathology. Here there is an account of finding submicroscopic deletions in $70 \%$ of patients with Duchenne or Becker muscular dystrophy, a discussion of germline mosaicism, or the presence of a microinversion in a female to explain multiple cases born to non-carrier parents, and a warning that such rare families must produce caution when giving genetic advice. There is an account in this chapter of the isolation of dystrophin with its implications for the pathogenesis of muscular dystrophy, but no mention of dystrophin in the chapter on confirmation of the diagnosis. These few useful additions do not warrant buying the revised edition as well as the first, but they ensure that the book remains a thoroughly sound practical, and valuable monograph of widespread interest.

SARAh BUndey 